\title{
Prognostic significance of sarcopenia in patients with hepatocellular carcinoma undergoing sorafenib therapy
}

\author{
HIROKI NISHIKAWA $^{1 *}$, NORIHIRO NISHIJIMA ${ }^{2 *}$, HIRAYUKI ENOMOTO ${ }^{1}$, AZUSA SAKAMOTO ${ }^{2}$, \\ AKIHIRO NASU $^{2}$, HIDEYUKI KOMEKADO ${ }^{2}$, TAKASHI NISHIMURA ${ }^{1}$, RYUICHI KITA ${ }^{2}$, \\ TORU KIMURA $^{2}$, HIROKO IIJIMA ${ }^{1}$, SHUHEI NISHIGUCHI ${ }^{1}$ and YUKIO OSAKI $^{2}$ \\ ${ }^{1}$ Division of Hepatobiliary and Pancreatic Disease, Department of Internal Medicine, \\ Hyogo College of Medicine, Nishinomiya, Hyogo 663-8501; ${ }^{2}$ Department of Gastroenterology \\ and Hepatology, Osaka Red Cross Hospital, Osaka 543-8555, Japan
}

Received July 14, 2016; Accepted March 17, 2017

DOI: $10.3892 / 01.2017 .6287$

\begin{abstract}
The present study aimed to examine the impact of sarcopenia, defined as low muscle mass on computed tomography (CT), prior to sorafenib therapy on the clinical outcomes of patients with hepatocellular carcinoma (HCC) receiving sorafenib therapy. In total, 232 patients with unresectable HCC (median age, 72 years) were analyzed, and the extent of sarcopenia was assessed using CT. Cross-sectional areas $\left(\mathrm{cm}^{2}\right)$ of the skeletal muscles at the third lumbar vertebra level were determined by manual outlining on the CT images. The cross-sectional areas were normalized for height [skeletal muscle index $\left.(\mathrm{SMI}), \mathrm{cm}^{2} / \mathrm{m}^{2}\right]$. Based on the findings of previous studies, male patients with SMI $\leq 36.2 \mathrm{~cm}^{2} / \mathrm{m}^{2}$ and female patients with SMI $\leq 29.6 \mathrm{~cm}^{2} / \mathrm{m}^{2}$ were defined as having
\end{abstract}

Correspondence to: Dr Hirayuki Enomoto, Division of Hepatobiliary and Pancreatic Disease, Department of Internal Medicine, Hyogo College of Medicine, 1-1 Mukogawacho, Nishinomiya, Hyogo 663-8501, Japan

E-mail: enomoto@hyo-med.ac.jp

*Contributed equally

Abbreviations: HCC, hepatocellular carcinoma; LC, liver cirrhosis; CT, dynamic computed tomography; TACE, transcatheter arterial chemoembolization; TAI, transcatheter arterial infusion chemotherapy; PS, performance status; ECOG, Eastern Cooperative Oncology Group; HU, Hounsfield unit; L3, third lumbar vertebra; SMI, skeletal muscle index; OS, overall survival; PFS, progression-free survival; SAE, serious adverse event; mRECIST, modified Response Evaluation Criteria in Solid Tumors; CR, complete response; $\mathrm{PR}$, partial response; $\mathrm{SD}$, stable disease; $\mathrm{PD}$, progressive disease; ORR, objective response rate; DCR, disease control rate; CTCAE, Common Terminology Criteria for Adverse Events; AST, aspartate aminotransferase; DCP, des-on Termxy prothrombin; NE, not evaluated; HR, hazard ratio; CI, confidence interval

Key words: hepatocellular carcinoma, sarcopenia, sorafenib, overall survival, progression-free survival, prognostic impact sarcopenia. The baseline characteristics, overall survival (OS) rates, progression-free survival (PFS) rates and best treatment response of the sarcopenia group were retrospectively compared with those of the non-sarcopenia group, and the factors associated with OS and PFS were examined. Sarcopenia was observed in 151 patients (65.1\%). There were 165 patients with Child-Pugh A and 67 with Child-Pugh B cirrhosis. In the sarcopenia group, the median treatment duration was 66 days, whereas in the non-sarcopenia group it was 103 days $(\mathrm{P}=0.001)$. The median OS time was 174 days in the sarcopenia group and 454 days in the non-sarcopenia group $(\mathrm{P}<0.0001)$. The median PFS was 77 days in the sarcopenia group and 106 days in the non-sarcopenia group $(\mathrm{P}=0.0131)$. Multivariate analysis identified sarcopenia to be an independent predictor of OS (hazard ratio, $0.365 ; \mathrm{P}<0.0001)$. The objective response rate and disease control rate in the sarcopenia group were significantly lower, compared with those in the non-sarcopenia group $(\mathrm{P}=0.0146$ and $\mathrm{P}=0.0151$, respectively). In conclusion, sarcopenia may be an indicator of poor clinical course in patients with HCC receiving sorafenib.

\section{Introduction}

Hepatocellular carcinoma (HCC) is one of the major causes of cancer-associated mortality worldwide, accounting for $5.7 \%$ of all newly diagnosed malignancies (1-5). The annual incidence rates of HCC are the highest in East Asia and Sub-Saharan Africa, where $>80 \%$ of all known cases develop (1-5). Advances in treatments for HCC during the last few decades markedly improved the prognosis of the disease $(1,2,4,5)$. However, a curative therapy such as surgical resection may be applied to a limited number $(<20 \%)$ of patients with $\operatorname{HCC}(5,6)$.

Sorafenib is a multi-kinase inhibitor that suppresses cancer growth and cell proliferation $(7,8)$. Two pivotal randomized phase III studies, namely the Sorafenib HCC Assessment Randomized Protocol study (7) and the Asian Pacific study (8), demonstrated that patients with unresectable HCC undergoing sorafenib therapy had significantly longer survival time compared with the placebo group. Although $>5$ years have elapsed since the introduction of sorafenib for the treatment of unresectable HCC in daily clinical practice, 
sorafenib is still regarded as first-line systemic chemotherapeutic agent for HCC (9-11). In addition, studies on prognostic factors in patients with HCC who underwent sorafenib therapy have mainly focused on tumor-associated factors, liver function, serum biomarkers and combination therapy with sorafenib (12-17).

Substantial skeletal muscle wasting, termed sarcopenia, is an important predictor for survival in patients with solid malignancies (18). By contrast, sarcopenia has become a relevant clinical feature for understanding the effects of aging on clinical outcomes (19). Sarcopenia is a commonly observed disorder in aged populations and is associated with disability, functional decline and frailty $(19,20)$. Generally, skeletal muscle mass is regulated depending on the balance between protein synthesis and protein breakdown (21). Patients with HCC often have underlying liver cirrhosis (LC), and skeletal muscle loss is a major characteristic of protein energy malnutrition in patients with LC $(22,23)$. Age-associated sarcopenia is defined as primary sarcopenia, whereas LC is one of the causes of secondary sarcopenia $(19,22,23)$. Although commonly observed, malnutrition is frequently underdiagnosed or overlooked, and it is poorly characterized in patients with HCC and LC complications (21-23). In addition, to the best of our knowledge, although sarcopenia has been reported to be an adverse predictor in patients with HCC who may have a potential for curative therapy such as surgical resection, as well as in patients with several malignancies other than HCC, there have been no studies regarding the impact of sarcopenia on clinical outcomes in patients with HCC undergoing sorafenib therapy $(18,24-27)$. Therefore, it is imperative to address these issues. The aim of the present study was to examine the impact of sarcopenia prior to sorafenib therapy on the clinical outcomes in patients with HCC receiving sorafenib.

\section{Materials and methods}

Patients and indications of sorafenib treatment. Between June 2009 and August 2015, 234 patients with HCC treated with sorafenib (median age $=72$ years, range; 40-91 years, 182 males and 52 females) were admitted to the Division of Hepatobiliary and Pancreatic Disease, Department of Internal Medicine, Hyogo College of Medicine (Hyogo, Japan) and the Department of Gastroenterology and Hepatology, Osaka Red Cross Hospital (Osaka, Japan). Of these, two patients with insufficient clinical data were excluded from the analysis. Thus, 232 patients with HCC treated with sorafenib were analyzed in the present study. The majority of analyzed patients received previous therapies for HCC. Sorafenib therapy was recommended for patients with unresectable $\mathrm{HCC}$ and the following features, as determined by dynamic computed tomography (CT): i) The presence of distant metastases; ii) refractory response to previous transcatheter arterial therapies for HCC [transcatheter arterial chemoembolization (TACE) or transcatheter arterial infusion (TAI) chemotherapy]; iii) unsuitability for TACE or TAI due to anatomical reasons; iv) vascular invasion such as tumor thrombus in the portal vein (28-30). Patients with poor performance status [PS; Eastern Cooperative Oncology Group (ECOG) classification $\geq 3$ ] were not recommended for sorafenib therapy $(28,29)$.

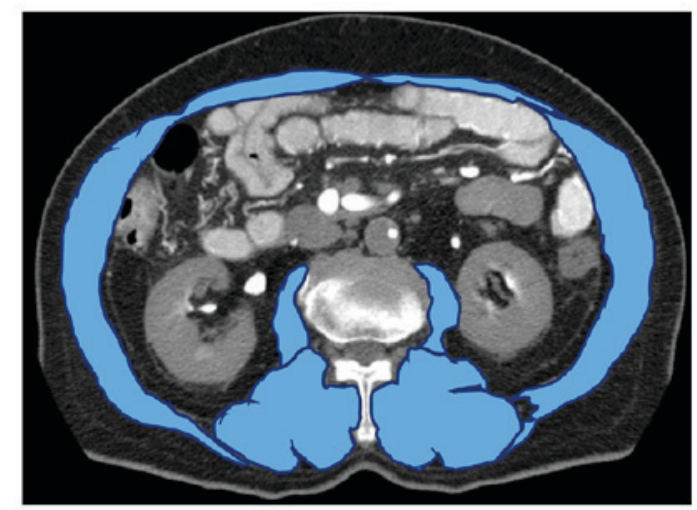

Figure 1. CT scan of a representative case. Cross-sectional areas $\left(\mathrm{cm}^{2}\right)$ of skeletal muscles at the third lumbar level were measured by manual tracing on the CT images, and their sum was calculated. The blue area is showing skeletal muscle at the third lumbar level. CT, computed tomography.

Definition of sarcopenia and the study protocol. Assessment of sarcopenia was performed using CT scans obtained prior to sorafenib therapy. The tissue Hounsfield unit (HU) limit for skeletal muscles on the CT image was $-29 \mathrm{HU}$ to $+150 \mathrm{HU}$, as previously reported (27). The third lumbar vertebra (L3) was used as a standard landmark. Skeletal muscles at the L3 level included the erector spinae, transverse abdominis, psoas, quadratus lumborum, internal and external oblique abdominal muscle and the rectus abdominis muscle; these muscles were identified on the CT images. Cross-sectional areas $\left(\mathrm{cm}^{2}\right)$ of the muscles were measured by manual tracing on the $\mathrm{CT}$ images, and their sum was calculated (27). A representative case is presented in Fig. 1. The cross-sectional areas were normalized for patient height [skeletal muscle index (SMI), $\mathrm{cm}^{2} / \mathrm{m}^{2}$ ]. Male patients with SMI $\leq 36.2 \mathrm{~cm}^{2} / \mathrm{m}^{2}$ and female patients with SMI $\leq 29.6 \mathrm{~cm}^{2} / \mathrm{m}^{2}$ were defined as having sarcopenia, based on the findings of a previous study (31).

The present study retrospectively compared baseline characteristics, overall survival (OS), progression-free survival (PFS), best treatment response of sorafenib and serious adverse events [SAEs; grade $\geq 3$ as defined by the Common Terminology Criteria for Adverse Events (CTCAE); version 3 (32)] in the sarcopenia and the non-sarcopenia groups, and investigated factors associated with OS and PFS using univariate and multivariate analysis. The current study was performed in accordance with the Declaration of Helsinki and with approval from the Ethics Committees of each hospital (Hyogo College of Medicine and Osaka Red Cross Hospital). The requirement to obtain written informed consent for inclusion in the present study from patients was waived.

HCC diagnosis and sorafenib therapy. HCC was diagnosed according to the previously described methods $(28,29)$. Briefly, dynamic CT of the liver was performed prior to initiating sorafenib therapy. For patients with atypical imaging findings, ultrasound-guided tumor biopsy was conducted for histological assessment. HCC was finally diagnosed based on radiological or histological findings in accordance with the guidelines of the European Association for the Study of the Liver (33).

For patients with no evident risk factors, the recommended initial dose of $800 \mathrm{mg} /$ day of sorafenib (400 mg 
Table I. Baseline characteristics $(n=232)$.

\begin{tabular}{lc}
\hline Variables & $\begin{array}{c}\text { Number of patients or } \\
\text { median value, } n \text { (range) }\end{array}$
\end{tabular}

Age, years

$72(40-91)$

Sarcopenia

Yes

No

Gender

Male

Female

Causes of liver disease

B

C

Non-B/non-C

49

$\mathrm{B}$ and $\mathrm{C}$

Unknown

Initial dose of sorafenib, mg/day

800

600

400

200

Child-Pugh

A

B

ECOG performance status

0

1

2

HCC stage

I

II

III

IVA

IVB

Previous therapies for $\mathrm{HCC}$

Transcatheter arterial therapies

Yes

No

Percutaneous ablative therapies

Yes

No

Surgical resection

Yes

No

Tumor burden $\geq 50 \%$

Yes

No
Table I. Continued.

Variables

Number of patients or

Variables
median value, $\mathrm{n}$ (range)

Total bilirubin, mg/dl

$0.8(0.2-5.1)$

Serum albumin, g/dl

$3.4(1.7-4.8)$

Prothrombin time, \%

$80(48-116)$

Platelets, x104/mm3

$11.7(3.4-56.7)$

AST, IU/l

$50(15-791)$

ALT, IU/1

34 (6-380)

ALP, IU/1

$401(124-4,535)$

GGT, IU/1 ${ }^{\mathrm{a}}$

$72(14-2,172)$

AFP, ng/ml ${ }^{b}$

$139.2(1.7-688,400)$

DCP, $\mathrm{mAU} / \mathrm{ml}^{\mathrm{c}}$

$748(10-421,210)$

Data are expressed as the number of patients or the or median (range). ECOG, the Eastern Cooperative Oncology Group; HCC, hepatocellular carcinoma; AST, aspartate aminotransferase; ALT, alanine aminotransferase; ALP, alkaline phosphatase; GGT, $\gamma$-glutamyl transpeptidase; AFP, $\alpha$-fetoprotein; DCP, des-oproteinn prothrombin. ${ }^{\mathrm{a}}$ Missing data, $\mathrm{n}=3$; ${ }^{\mathrm{b}}$ missing data, $\mathrm{n}=1$; ${ }^{\mathrm{c}}$ missing data, $\mathrm{n}=3$.

twice a day) was administered $(7,8)$. The reduced initial dose was administered to a number of patients $(n=166)$ based on clinical features, including body weight, age, ECOG-PS and liver function. During sorafenib treatment, each attending physician adjusted the daily dose of sorafenib according to the degree of adverse events. In patients who received an initial reduced dose of sorafenib and exhibited good tolerability, dose escalation from 400-600 mg/day or from 400-800 mg/day was permitted. In patients with adverse events of grade $\geq 3$, sorafenib treatment was discontinued until the clinical symptoms resolved to grade 1 or 2 . In principle, the treatment efficacy of sorafenib was assessed every 4-8 weeks following the initiation of therapy, according to the modified Response Evaluation Criteria in Solid Tumors (mRECIST) and/or the levels of tumor markers $(28,29,34,35)$. Patients continued sorafenib until the development of the following conditions: Unacceptable sorafenib-associated toxicity, disease progression or the patient's wish to discontinue treatment. Following discontinuation of sorafenib therapy for any reason, physicians evaluated the clinical conditions (tumor status or the general status) of each patient and investigated the suitability of other therapies (TACE, TAI or systemic chemotherapy other than sorafenib) for achieving the best clinical outcome $(28,29)$.

Evaluation of treatment efficacy. The best treatment efficacy achieved during sorafenib therapy was determined according to the mRECIST criteria and/or tumor marker levels as previously indicated $(28,29,34)$. The treatment efficacy was classified into the following four categories: Complete response (CR); partial response (PR); stable disease (SD); progressive disease (PD). A patient with CR was characterized by the absence of enhancement in the arterial phase within all targeted nodules. A patient with PR was characterized by a $\geq 30 \%$ reduction in tumor size, which was determined by calculating the sum of the diameters of the targeted nodules. The size of the nodules 
A

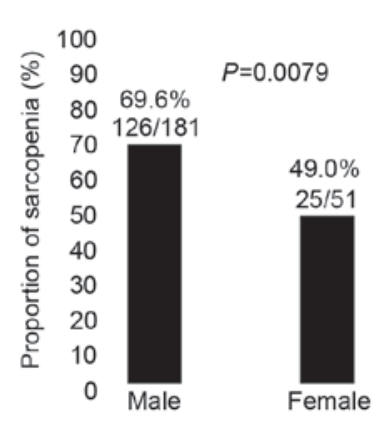

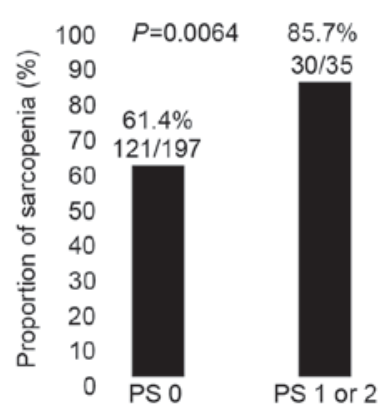

C

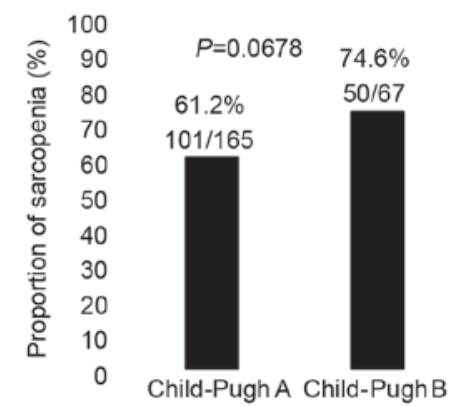

Figure 2. Comparison of the proportion of sarcopenia. (A) The proportion of sarcopenia in male patients as compared with that in female patients was significantly higher $(\mathrm{P}=0.0079)$. (B) The proportion of sarcopenia in patients with poorer the Eastern Cooperative Oncology Group-PS as compared with that in patients with PS-0 was significantly higher $(\mathrm{P}=0.0137) .(\mathrm{C})$ The proportion of sarcopenia in patients with Child-Pugh class A cirrhosis as compared with Child-Pugh class $\mathrm{B}$ cirrhosis tended to be lower $(\mathrm{P}=0.0678)$. PS, performance status.

was estimated via unidirectional measurement. A patient with PD was characterized by $a \geq 20 \%$ increase in the tumor size via calculating the sum of the maximal dimensions of the targeted nodules. A patient with SD was characterized by the absence of CR, PR or PD $(29,34)$. The objective response rate (ORR) was defined as the percentage of patients with the best tumor response rates considering $\mathrm{CR}$ and $\mathrm{PR}$. The disease control rate (DCR) was defined as the percentage of patients with the best tumor response rates considering $\mathrm{CR}, \mathrm{PR}$ and SD.

Safety evaluation of sorafenib therapy. Sorafenib associated adverse events, including rash, diarrhea, hand-foot skin reaction, hypertension, liver damage, fatigue, gastrointestinal hemorrhage and lung injury, were evaluated using CTCAE version 3.0 (32).

Statistical analysis. The categorical variables of the sarcopenia and non-sarcopenia groups were analyzed by Fisher's exact test, while the numerical variables were analyzed with the unpaired Student's t-test or with the Mann-Whitney U test as applicable. OS and PFS curves were generated using the Kaplan-Meier method and compared using the log-rank test. Factors with values of $\mathrm{P}<0.05$ in univariate analysis were included in the multivariate analysis with the Cox proportional hazards model. In order to analyze the significance of predictors in multivariate analysis, numerous variables were divided by the median values for all cases $(n=232)$ and treated as dichotomous covariates. OS was defined as the period from the initiation of sorafenib therapy until mortality (due to any cause) or the last follow-up visit. PFS was defined as the period from the initiation of sorafenib therapy until the date of the detection of progression-free disease or mortality (due to any cause) $(28,29)$. Data are expressed as median values (range). $\mathrm{P}<0.05$ was considered to indicate a statistically significant difference. All statistical analyses were performed using the JMP 11 software (SAS Institute, Inc., Cary, NC, USA).

\section{Results}

Baseline characteristics. The baseline characteristics of the patients $(n=232)$ are presented in Table I. There were 181 male and 51 female patients with a median age of 72 years (range,
40-91). Sarcopenia was observed in 151 (65.1\%) patients. There were 165 patients with Child-Pugh class A and 67 patients with Child-Pugh class B cirrhosis (36). In 66 (28.4\%) patients, the standard dose of sorafenib ( $800 \mathrm{mg} /$ day) was administered at the beginning of therapy. Previously, the most common therapies for were transcatheter arterial therapies, including TACE or TAI, followed by percutaneous ablative therapies and surgical resection.

Comparison of baseline characteristics between patients with and without sarcopenia. Compared with those in the non-sarcopenia group, the proportion of sarcopenia in male patients as compared with that in female patients was significantly higher $(\mathrm{P}=0.0079$; Fig. 2A) and the proportion of sarcopenia in patients with poorer ECOG-PS as compared with that in patients with PS-0 was significantly higher $(\mathrm{P}=0.0137$; Fig. $2 \mathrm{~B})$, whereas the proportion of sarcopenia in patients with Child-Pugh class A cirrhosis as compared with Child-Pugh class B cirrhosis tended to be lower $(\mathrm{P}=0.0678$; Fig. $2 \mathrm{C})$ and patients treated with an initial sorafenib dose of $800 \mathrm{mg} /$ day $(\mathrm{P}=0.096)$ in the sarcopenia group tended to be significantly lower compared with those in the non-sarcopenia group (Table II). Laboratory analysis revealed that the differences between the sarcopenia and non-sarcopenia groups were significant with regard to the levels of serum albumin $(\mathrm{P}=0.0022)$, aspartate aminotransferase (AST; $\mathrm{P}=0.0062)$ and des- $\gamma$-carboxy prothrombin (DCP; $\mathrm{P}=0.0007$; Table II).

Comparison of OS and PFS rates between patients with and without sarcopenia. The median follow-up periods subsequent to sorafenib treatment were 170 days (range, 12-1,145) in the sarcopenia group and 419 days (range, 50-2,036) in the non-sarcopenia group. The median OS was 174 days in the sarcopenia group and 454 days in the non-sarcopenia group $(\mathrm{P}<0.0001$; Fig. 3A). The median PFS was 77 days in the sarcopenia group and 106 days in the non-sarcopenia group $(\mathrm{P}=0.0131$; Fig. 3B).

Comparison of treatment duration and SAEs of grade $\geq 3$ between patients with and without sarcopenia. The median treatment duration was 66 days in the sarcopenia group, and 103 days in the non-sarcopenia group $(\mathrm{P}=0.001)$. The prevalence of sorafenib-associated SAEs of grade $\geq 3$, as 
Table II. Comparisons between patients with and without sarcopenia.

\begin{tabular}{|c|c|c|c|}
\hline Variables & Sarcopenia, n (range) & Non-sarcopenia, n (range) & P-value \\
\hline Total & 151 & 81 & \\
\hline Age, years & $72(46-91)$ & $71(40-85)$ & 0.1456 \\
\hline Gender & & & 0.0079 \\
\hline Male & 126 & 55 & \\
\hline Female & 25 & 26 & \\
\hline Causes of liver disease & & & 0.6426 \\
\hline $\mathrm{B}$ & 22 & 11 & \\
\hline $\mathrm{C}$ & 93 & 52 & \\
\hline Non-B/non-C & 31 & 17 & \\
\hline $\mathrm{B}$ and $\mathrm{C}$ & 4 & 0 & \\
\hline Unknown & 1 & 1 & \\
\hline Child-Pugh, A/B & & & 0.0678 \\
\hline A & 101 & 64 & \\
\hline B & 50 & 17 & \\
\hline ECOG performance status & & & 0.0137 \\
\hline 0 & 121 & 76 & \\
\hline 1 & 26 & 4 & \\
\hline 2 & 4 & 1 & \\
\hline Initial dose of sorafenib, mg/day & & & 0.0960 \\
\hline 800 & 37 & 29 & \\
\hline 600 & 0 & 1 & \\
\hline 400 & 112 & 50 & \\
\hline 200 & 2 & 1 & \\
\hline HCC stage & & & 0.3353 \\
\hline I & 1 & 0 & \\
\hline II & 10 & 8 & \\
\hline III & 48 & 31 & \\
\hline IVA & 35 & 11 & \\
\hline IVB & 57 & 31 & \\
\hline Tumor burden $\geq 50 \%$ & & & 0.4900 \\
\hline Yes & 17 & 6 & \\
\hline No & 134 & 75 & \\
\hline Total bilirubin, mg/dl & $0.8(0.3-2.5)$ & $0.8(0.2-5.1)$ & 0.4279 \\
\hline Serum albumin, g/dl & $3.4(1.7-4.8)$ & $3.5(2.0-4.8)$ & 0.0022 \\
\hline Prothrombin time, $\%$ & $79(48-116)$ & $81(60-111)$ & 0.4466 \\
\hline Platelets, $x 10^{4} / \mathrm{mm}^{3}$ & $11.6(3.4-47.9)$ & $11.8(3.6-56.7)$ & 0.8461 \\
\hline $\mathrm{AST}, \mathrm{IU} / 1$ & $55(15-791)$ & $43(17-679)$ & 0.0062 \\
\hline ALT, IU/1 & $36(6-380)$ & $30(9-290)$ & 0.8302 \\
\hline ALP, IU/1 & $429(161-4535)$ & $391(124-3,265)$ & 0.6496 \\
\hline GGT, IU/1 ${ }^{\mathrm{a}}$ & $79.5(15-941)$ & $68(14-2,172)$ & 0.2377 \\
\hline $\mathrm{AFP}, \mathrm{ng} / \mathrm{ml}^{\mathrm{b}}$ & $138.3(1.8-688,400)$ & $162.9(1.7-98,435)$ & 0.7965 \\
\hline $\mathrm{DCP}, \mathrm{mAU} / \mathrm{ml}^{\mathrm{c}}$ & $1,305(10-421,210)$ & $292.5(10-53,857)$ & 0.0007 \\
\hline Serious adverse events, grade $\geq 3$ & $41.1 \%(62 / 151)$ & $33.3 \%(27 / 81)$ & 0.2610 \\
\hline Best treatment response & & & 0.0185 \\
\hline $\mathrm{CR}$ & 1 & 3 & \\
\hline PR & 5 & 8 & \\
\hline SD & 40 & 27 & \\
\hline $\mathrm{PD}$ & 61 & 30 & \\
\hline $\mathrm{NE}$ & 44 & 13 & \\
\hline
\end{tabular}


Table II. Continued.

\begin{tabular}{lccc}
\hline Variables & Sarcopenia, n (range) & Non-sarcopenia, n (range) & P-value \\
\hline Objective response rate & $4.0 \%(6 / 151)$ & $13.6 \%(11 / 81)$ & 0.0146 \\
Disease control rate & $30.5 \%(46 / 151)$ & $46.9 \%(38 / 81)$ & 0.0151 \\
\hline
\end{tabular}

Data are presented as the number of patients or the median value (range). ECOG, the Eastern Cooperative Oncology Group; HCC, hepatocellular carcinoma; AST, aspartate aminotransferase; ALT, alanine aminotransferase; ALP, alkaline phosphatase; GGT, $\gamma$-glutamyl transpeptidase; AFP, $\alpha$-fetoprotein; DCP, des-oproteinn prothrombin; CR, complete response; PR, partial response; SD, stable disease; PD, progressive disease; NE, not evaluated. ${ }^{a}$ Missing data, $n=3 ;{ }^{b}$ missing data, $n=1 ;{ }^{c}$ missing data, $n=3$.

A

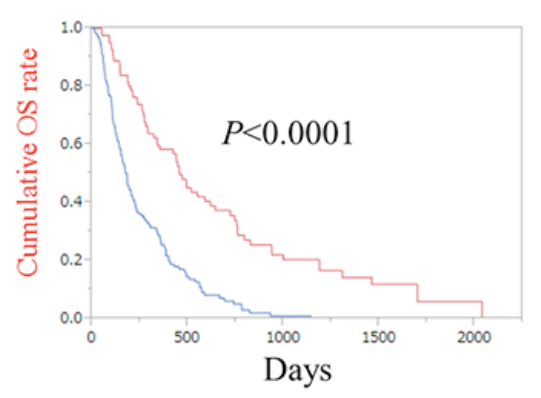

B

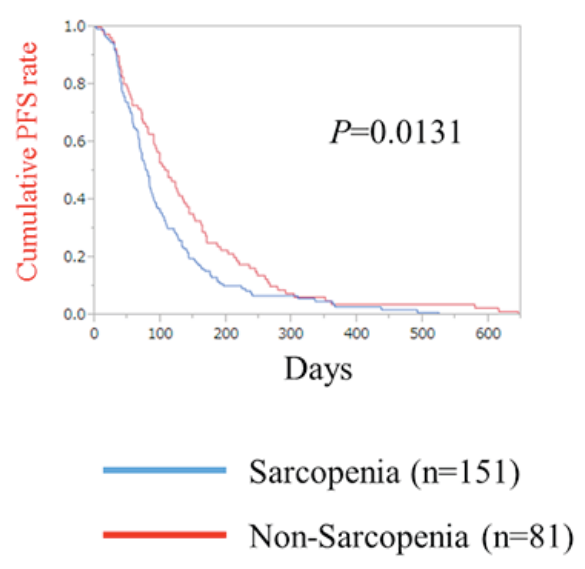

Figure 3. Kaplan-Meier curves showing cumulative OS and PFS rates in the sarcopenia and non-sarcopenia groups. (A) The median OS was 174 days in the sarcopenia group and 454 days in the non-sarcopenia group $(\mathrm{P}<0.0001)$. (B) The median PFS was 77 days in the sarcopenia group and 106 days in non-sarcopenia group $(\mathrm{P}=0.0131)$. OS, overall survival; PFS, progression-free survival.

assessed by CTCAE version 3.0 , was $41.1 \%(62 / 151)$ in the sarcopenia group and $33.3 \%(27 / 81)$ in the non-sarcopenia group $(\mathrm{P}=0.261)$.

Best tumor treatment response in the sarcopenia and non-sarcopenia groups. In the analysis of the best tumor response in the sarcopenia group, CR was achieved in 1 patient, $\mathrm{PR}$ in 5, SD in 40 and PD in 61, while 44 were not evaluated (NE); the ORR and DCR were calculated to be $4.0 \%(6 / 151)$ and $30.5 \%(46 / 151)$, respectively. In the analysis of the best tumor response in the non-sarcopenia group, CR was achieved in 3 patients, $\mathrm{PR}$ in $8, \mathrm{SD}$ in $27, \mathrm{PD}$ in 30 and 13 were $\mathrm{NE}$; the ORR and DCR were calculated to be $13.6 \%$ (11/81) and $46.9 \%$ (38/81), respectively. The best treatment efficacy significantly differed between the sarcopenia and non-sarcopenia groups (ORR, P=0.0146; DCR, $\mathrm{P}=0.0151$; Table II).

Causes of mortality. In the sarcopenia group, $136(90.1 \%)$ patients expired during the follow-up period: 111 due to HCC progression; 6 of liver failure; 19 of other causes. In the non-sarcopenia group, $63(77.8 \%)$ patients perished during the follow-up period: 60 due to HCC progression; 1 of liver failure; 2 of other causes.

Univariate and multivariate analysis of factors contributing to $O S$. The univariate analysis identified that the following factors significantly contributed to OS for all cases $(n=232)$ : Sex $(\mathrm{P}=0.0079)$; initial dose of sorafenib $(\mathrm{P}=0.0394)$; sarcopenia $(\mathrm{P}<0.0001)$; ECOG-PS $(\mathrm{P}=0.0041)$; extrahepatic metastases $(\mathrm{P}=0.0024)$; portal vein invasion $(\mathrm{P}=0.0029)$; tumor burden $\geq 50 \% \quad(\mathrm{P}=0.0001)$; presence of ascites $(\mathrm{P}<0.0001) ; \mathrm{AST} \geq 50 \mathrm{IU} / 1(\mathrm{P}=0.0081)$; alkaline phosphatase $\geq 401 \mathrm{IU} / \mathrm{l}(\mathrm{P}=0.0301)$; serum albumin $\geq 3.4 \mathrm{~g} / \mathrm{dl}(\mathrm{P}=0.0010)$; $\alpha$-fetoprotein $\geq 139.2 \mathrm{ng} / \mathrm{ml}(\mathrm{P}=0.0286) ; \mathrm{DCP} \geq 748 \mathrm{mAU} / \mathrm{ml}$ $(\mathrm{P}=0.0037$; Table III). The hazard ratios (HRs) and $95 \%$ confidence intervals (CIs) determined by multivariate analysis for the 13 variables (selected based on $\mathrm{P}<0.05$ values in univariate analysis) are detailed in Table III. Using multivariate analysis, sarcopenia $(\mathrm{P}<0.0001)$, extrahepatic metastases $(\mathrm{P}<0.0001)$, tumor burden $\geq 50 \% \quad(\mathrm{P}=0.0004)$ and the presence of ascites $(\mathrm{P}=0.0002)$ were identified to be significant predictors of OS.

Univariate and multivariate analysis of factors contributing to PFS. Univariate analysis identified sarcopenia $(\mathrm{P}=0.0131)$, ECOG-PS $(\mathrm{P}=0.0021)$, extrahepatic metastases $(\mathrm{P}=0.0019)$, portal vein invasion $(\mathrm{P}=0.0203)$, tumor burden $\geq 50 \%$ $(\mathrm{P}=0.0244)$, presence of ascites $(\mathrm{P}=0.0429)$ and $\mathrm{DCP}$ $\geq 748 \mathrm{mAU} / \mathrm{ml}(\mathrm{P}=0.0266)$ to be significantly associated with PFS for all cases $(n=232$; Table IV). The HRs and 95\% CIs determined by multivariate analysis for these seven factors (selected based on $\mathrm{P}<0.05$ values in univariate analysis) 
Table III. Univariate and multivariate analysis of factors contributing to overall survival.

\begin{tabular}{|c|c|c|c|c|}
\hline \multirow[b]{2}{*}{ Variables } & \multirow[b]{2}{*}{ Patients, $\mathrm{n}$} & \multirow[b]{2}{*}{ Univariate analysis } & \multicolumn{2}{|c|}{ Multivariate analysis } \\
\hline & & & Hazard ratio $(95 \% \mathrm{CI})$ & P-value \\
\hline Gender & & 0.0079 & $0.736(0.477-1.111)$ & 0.1464 \\
\hline Male & 181 & & & \\
\hline Female & 51 & & & \\
\hline Age, years & & 0.5742 & & \\
\hline$\geq 72$ & 119 & & & \\
\hline$<72$ & 113 & & & \\
\hline Initial dose of sorafenib & & 0.0394 & $1.072(0.750-1.547)$ & 0.7068 \\
\hline $800 \mathrm{mg} / \mathrm{day}$ & 66 & & & \\
\hline Reduced dose of sorafenib & 166 & & & \\
\hline Sarcopenia & & $<0.0001$ & $0.365(0.255-0.516)$ & $<0.0001$ \\
\hline Yes & 151 & & & \\
\hline No & 81 & & & \\
\hline ECOG-PS 0 & & 0.0041 & $1.098(0.717-1.636)$ & 0.6581 \\
\hline Yes & 197 & & & \\
\hline No & 35 & & & \\
\hline Extrahepatic metastases & & 0.0024 & $0.523(0.383-0.715)$ & $<0.0001$ \\
\hline Yes & 88 & & & \\
\hline No & 144 & & & \\
\hline Portal vein invasion & & 0.0229 & $0.734(0.521-1.051)$ & 0.0900 \\
\hline Yes & 52 & & & \\
\hline No & 180 & & & \\
\hline Tumor burden $\geq 50 \%$ & & 0.0001 & $0.357(0.218-0.614)$ & 0.0004 \\
\hline Yes & 23 & & & \\
\hline No & 209 & & & \\
\hline Ascites & & $<0.0001$ & $0.427(0.283-0.715)$ & 0.0002 \\
\hline Yes & 195 & & & \\
\hline No & 37 & & & \\
\hline AST, IU/l & & 0.0081 & $0.774(0.564-1.061)$ & 0.1116 \\
\hline$\geq 50$ & 121 & & & \\
\hline$<50$ & 111 & & & \\
\hline ALT, IU/1 & & 0.0833 & & \\
\hline$\geq 34$ & 117 & & & \\
\hline$<34$ & 115 & & & \\
\hline ALP, IU/1 & & 0.0301 & $0.993(0.724-1.356)$ & 0.9643 \\
\hline$\geq 401$ & 116 & & & \\
\hline$<401$ & 116 & & & \\
\hline GGT, IU/1 & & 0.0823 & & \\
\hline$\geq 72$ & 115 & & & \\
\hline$<72$ & 114 & & & \\
\hline Prothrombin time, $\%$ & & 0.1215 & & \\
\hline$\geq 80$ & 117 & & & \\
\hline$<80$ & 115 & & & \\
\hline Serum albumin level, g/dl & & 0.0010 & $1.160(0.827-1.622)$ & 0.3879 \\
\hline$\geq 3.4$ & 127 & & & \\
\hline$<3.4$ & 105 & & & \\
\hline Total bilirubin, $\mathrm{mg} / \mathrm{dl}$ & & 0.1166 & & \\
\hline$\geq 0.8$ & 129 & & & \\
\hline$<0.8$ & 103 & & & \\
\hline
\end{tabular}


Table III. Continued.

\begin{tabular}{|c|c|c|c|c|}
\hline \multirow[b]{2}{*}{ Variables } & \multirow[b]{2}{*}{ Patients, $\mathrm{n}$} & \multirow[b]{2}{*}{ Univariate analysis } & \multicolumn{2}{|c|}{ Multivariate analysis } \\
\hline & & & Hazard ratio $(95 \% \mathrm{CI})$ & P-value \\
\hline Platelet count, $\times 10^{4} / \mathrm{mm}^{3}$ & & 0.5146 & & \\
\hline$\geq 11.7$ & 116 & & & \\
\hline$<11.7$ & 116 & & & \\
\hline Serum AFP, ng/ml & & 0.0286 & $0.743(0.542-1.015)$ & 0.0619 \\
\hline$\geq 139.2$ & 116 & & & \\
\hline$<139.2$ & 115 & & & \\
\hline $\mathrm{DCP}, \mathrm{mAU} / \mathrm{ml}$ & & 0.0037 & $0.858(0.622-1.182)$ & 0.3492 \\
\hline$\geq 748$ & 115 & & & \\
\hline$<748$ & 114 & & & \\
\hline
\end{tabular}

CI, confidence interval; ECOG-PS, the Eastern Cooperative Oncology Group performance status; AST, aspartate aminotransferase; ALT, alanine aminotransferase; ALP, alkaline phosphatase; GGT, $\gamma$ glutamyl transpeptidase; AFP, $\alpha$-fetoprotein; DCP, des-oprotein; prothrombin. ${ }^{\mathrm{a} C o x}$ proportional hazard model.

Table IV. Univariate and multivariate analysis of factors contributing to progression-free survival.

\begin{tabular}{|c|c|c|c|c|}
\hline \multirow[b]{2}{*}{ Variables } & \multirow[b]{2}{*}{ Patients, $\mathrm{n}$} & \multirow[b]{2}{*}{ Univariate analysis } & \multicolumn{2}{|c|}{ Multivariate analysis } \\
\hline & & & Hazard ratio $(95 \% \mathrm{CI})$ & P-value \\
\hline Gender & & 0.3319 & & \\
\hline Male & 181 & & & \\
\hline Female & 51 & & & \\
\hline Age, years & & 0.7418 & & \\
\hline$\geq 72$ & 119 & & & \\
\hline$<72$ & 113 & & & \\
\hline Initial dose of sorafenib & & 0.1065 & & \\
\hline $800 \mathrm{mg} / \mathrm{day}$ & 66 & & & \\
\hline Reduced dose of sorafenib & 166 & & & \\
\hline Sarcopenia & & 0.0131 & $0.831(0.612-1.123)$ & 0.2300 \\
\hline Yes & 151 & & & \\
\hline No & 81 & & & \\
\hline ECOG-PS 0 & & 0.0021 & $1.509(1.009-2.192)$ & 0.0452 \\
\hline Yes & 197 & & & \\
\hline No & 35 & & & \\
\hline Extrahepatic metastases & & 0.0019 & $0.627(0.475-0.833)$ & 0.0014 \\
\hline Yes & 88 & & & \\
\hline No & 144 & & & \\
\hline Portal vein invasion & & 0.0203 & $0.715(0.516-1.007)$ & 0.0547 \\
\hline Yes & 52 & & & \\
\hline No & 180 & & & \\
\hline Tumor burden $\geq 50 \%$ & & 0.0244 & $0.686(0.441-1.118)$ & 0.1255 \\
\hline Yes & 23 & & & \\
\hline No & 209 & & & \\
\hline Ascites & & 0.0429 & $0.695(0.485-1.025)$ & 0.0656 \\
\hline Yes & 195 & & & \\
\hline No & 37 & & & \\
\hline
\end{tabular}


Table IV. Continued.

\begin{tabular}{|c|c|c|c|c|}
\hline \multirow[b]{2}{*}{ Variables } & \multirow[b]{2}{*}{ Patients, $\mathrm{n}$} & \multirow[b]{2}{*}{ Univariate analysis } & \multicolumn{2}{|c|}{ Multivariate analysis } \\
\hline & & & Hazard ratio $(95 \% \mathrm{CI})$ & P-value ${ }^{a}$ \\
\hline AST, IU/1 & & 0.1455 & & \\
\hline$\geq 50$ & 121 & & & \\
\hline$<50$ & 111 & & & \\
\hline ALT, IU/1 & & 0.6526 & & \\
\hline$\geq 34$ & 117 & & & \\
\hline$<34$ & 115 & & & \\
\hline ALP, IU/1 & & 0.0977 & & \\
\hline$\geq 401$ & 116 & & & \\
\hline$<401$ & 116 & & & \\
\hline GGT, IU/1 & & 0.3614 & & \\
\hline$\geq 72$ & 115 & & & \\
\hline$<72$ & 114 & & & \\
\hline Prothrombin time, $\%$ & & 0.3787 & & \\
\hline$\geq 80$ & 117 & & & \\
\hline$<80$ & 115 & & & \\
\hline Serum albumin level, g/dl & & 0.1266 & & \\
\hline$\geq 3.4$ & 127 & & & \\
\hline$<3.4$ & 105 & & & \\
\hline Total bilirubin, mg/dl & & 0.6683 & & \\
\hline$\geq 0.8$ & 129 & & & \\
\hline$<0.8$ & 103 & & & \\
\hline Platelet count, $\times 10^{4} / \mathrm{mm}^{3}$ & & 0.1255 & & \\
\hline$\geq 11.7$ & 116 & & & \\
\hline$<11.7$ & 116 & & & \\
\hline Serum AFP, ng/ml & & 0.2879 & & \\
\hline$\geq 139.2$ & 116 & & & \\
\hline$<139.2$ & 115 & & & \\
\hline $\mathrm{DCP}, \mathrm{mAU} / \mathrm{ml}$ & & 0.0266 & $0.852(0.643-1.129)$ & 0.2641 \\
\hline$\geq 748$ & 115 & & & \\
\hline$<748$ & 114 & & & \\
\hline
\end{tabular}

CI, confidence interval; ECOG-PS, the Eastern Cooperative Oncology Group performance status; AST, aspartate aminotransferase; ALT, alanine aminotransferase; ALP, alkaline phosphatase; GGT, $\gamma$ glutamyl transpeptidase; AFP, $\alpha$-fetoprotein; DCP, des-oprotein; prothrombin. ${ }^{\mathrm{a} C}$ Cox proportional hazard model.

are presented in Table IV. Multivariate analysis identified ECOG-PS $(\mathrm{P}=0.0452)$ and extrahepatic metastasis $(\mathrm{P}=0.0014)$ to be significant prognostic factors associated with PFS.

\section{Discussion}

Recently, sarcopenia has attracted a high level of attention in the fields of several types of malignancies due to its impact on clinical outcomes $(18,24,25)$. However, to the best of our knowledge, reliable data regarding the impact of sarcopenia on the clinical outcomes of patients with $\mathrm{HCC}$ receiving sorafenib therapy have yet to be obtained. Therefore, the present study was conducted; to the best of our knowledge, it is the first study to evaluate the associations between sarcopenia and clinical outcomes in patients with unresectable $\mathrm{HCC}$ receiving sorafenib therapy. The major advantage of the current study was the large patient cohort.

Multivariate analysis identified sarcopenia to be an independent predictor of $\mathrm{OS}(\mathrm{HR}=0.365 ; \mathrm{P}<0.0001)$ and demonstrated its association with treatment efficacy. These results indicated that sarcopenia may be a significant predictor of prognosis in patients with HCC who underwent sorafenib therapy, and potentially in patients with other types of malignancies. Individualized nutritional assessment and interventional strategies may be recommended for patients with HCC and sarcopenia treated with sorafenib $(18,24,25)$. By contrast, it should be noted that sarcopenia was identified in $151(65.1 \%)$ patients in the present analysis. A potential 
explanation for the high prevalence is that the median age of the patients was 72 years. In Japan, the number of elderly patients with $\mathrm{HCC}$ has been increasing (5). These trends may be critical, as the incidence of sarcopenia in patients with HCC is predicted to increase in the future. Another possible reason is that, in the majority of cases, patients with HCC frequently underwent other treatments prior to sorafenib therapy. Popular HCC therapies may cause the deterioration of liver function, potentially leading to a decreased quality of life and the occurrence of sarcopenia in patients with HCC (37).

Consistent with previous studies, the presence of sarcopenia was associated with poor PS and poor liver function in the present study $(38,39)$. Furthermore, the ORR and DCR for the sarcopenia group were significantly lower than for the non-sarcopenia group. This may be attributed to the fact that the duration of treatment with sorafenib in the sarcopenia group was significantly shorter than that in the non-sarcopenia group ( $\mathrm{P}=0.001)$. Mir et al (40) reported that the presence of sarcopenia is associated with early dose-limiting toxicities and the pharmacokinetics of sorafenib in patients with HCC. These results are possibly associated with the results in the present study.

The recent increase in the prevalence of obesity has surfaced a novel clinical condition termed sarcopenic obesity, which is the combination of obesity and sarcopenia (41). As patients with cirrhosis develop sarcopenia even if they have obesity, a considerable number of cirrhotic patients are established to have sarcopenic obesity (41). Sarcopenic obesity has also been associated with poorer clinical outcomes in numerous types of malignancies (42). However, in the present study, the differences between the sarcopenia group with obesity (BMI $\left.\geq 25 \mathrm{~kg} / \mathrm{m}^{2} ; \mathrm{n}=21\right)$ and the sarcopenia group without obesity $(\mathrm{n}=130)$ were insignificant in terms of OS $(\mathrm{P}=0.8767)$ and PFS $(\mathrm{P}=0.2064$; data not presented) $(43,44)$. The reasons for this observation are unclear, and additional studies concerning the impact of sarcopenic obesity on the survival of patients with HCC treated with sorafenib are required.

Multivariate analysis identified the presence of extrahepatic metastasis as an independent predictor of OS $(\mathrm{HR}=0.523 ; \mathrm{P}<0.0001)$ and $\mathrm{PFS}(\mathrm{HR}=0.627 ; \mathrm{P}=0.0014)$. These results are consistent with the results from previous studies (7-9). Tumor-associated factors, including extrahepatic metastasis, maximum tumor size and vascular invasion, may potentially have the strongest prognostic impact on sorafenib therapy instead of liver function. In patients with HCC with significantly poor liver function, sorafenib therapy must be contraindicated $(7,8)$.

There are several limitations of the present study. First, it is a retrospective observational study. Second, the initial dose of sorafenib differed between the patients, creating bias. Third, various anticancer therapies were employed following the discontinuation of sorafenib, and these therapies may have potentially caused bias in the clinical outcomes of the patients. Fourth, certain data were missing in the analysis. However, owing to the small number of patients with missing data, this may not have affected the interpretation of the data. Finally, the present study population only included Japanese patients with relatively low body weights compared with patients in Western countries. Therefore, the present study results may not be directly applied to various ethnic populations. However, the results of the current study demonstrated that sarcopenia is associated with the clinical outcomes of patients with HCC undergoing sorafenib therapy. In conclusion, sarcopenia may be a significant predictor of prognosis in patients with HCC receiving sorafenib therapy. In such patients, appropriate interventions, such as nutritional therapies or exercise, may be required for improving the clinical outcomes.

\section{Acknowledgements}

The authors would like to thank Mrs. Haruko Takada (Osaka Red Cross Hospital), Mrs. Nozomi Kanazawa (Hyogo College of Medicine), Mrs. Yoko Matsushita (Hyogo College of Medicine) and Miss. Sayaka Fujii (Hyogo College of Medicine) for data collection.

\section{References}

1. El-Serag HB: Epidemiology of viral hepatitis and hepatocellular carcinoma. Gastroenterology 142: 1264-1273.e1, 2012.

2. El-Serag HB: Hepatocellular carcinoma. N Engl J Med 365: 1118-1127, 2011.

3. McGlynn KA, Petrick JL and London WT: Global epidemiology of hepatocellular carcinoma: An emphasis on demographic and regional variability. Clin Liver Dis 19: 223-238, 2015.

4. Singal AG and El-Serag HB: Hepatocellular carcinoma from epidemiology to prevention: Translating knowledge into practice. Clin Gastroenterol Hepatol 13: 2140-2151, 2015.

5. Osaki Y and Nishikawa H: Treatment for hepatocellular carcinoma in Japan over the last three decades: Our experience and literature review. Hepatol Res 45: 59-74, 2015.

6. Rahbari NN, Mehrabi A, Mollberg NM, Müller SA, Koch M, Büchler MW and Weitz J: Hepatocellular carcinoma: Current management and perspectives for the future. Ann Surg 253: 453-469, 2011.

7. Llovet JM, Ricci S, Mazzaferro V, Hilgard P, Gane E, Blanc JF, de Oliveira AC, Santoro A, Raoul JL, Forner A, et al: Sorafenib in advanced hepatocellular carcinoma. N Engl J Med 359: 378-390, 2008.

8. Cheng AL, Kang YK, Chen Z, Tsao CJ, Qin S, Kim JS, Luo R, Feng J, Ye S, Yang TS, et al: Efficacy and safety of sorafenib in patients in the Asia-Pacific region with advanced hepatocellular carcinoma: A phase III randomised, double-blind, placebo-controlled trial. Lancet Oncol 10: 25-34, 2009.

9. Di Marco V, De Vita F, Koskinas J, Semela D, Toniutto P and Verslype C: Sorafenib: From literature to clinical practice. Ann Oncol 24 (Suppl 2): ii30-ii37, 2013.

10. Sangiovanni A and Colombo M: Treatment of hepatocellular carcinoma: Beyond international guidelines. Liver Int 36 (Suppl 1): S124-S129, 2016.

11. Arizumi T, Ueshima K, Minami T, Kono M, Chishina H, Takita M, Kitai S, Inoue T, Yada N, Hagiwara S, et al: Effectiveness of sorafenib in patients with transcatheter arterial chemoembolization (TACE) refractory and intermediate-stage hepatocellular carcinoma. Liver Cancer 4: 253-226, 2015.

12. Bruix J, Raoul JL, Sherman M, Mazzaferro V, Bolondi L, Craxi A, Galle PR, Santoro A, Beaugrand M, Sangiovanni A, et al: Efficacy and safety of sorafenib in patients with advanced hepatocellular carcinoma: Subanalyses of a phase III trial. J Hepatol 57: 821-829, 2012.

13. Llovet JM, Peña CE, Lathia CD, Shan M, Meinhardt G and Bruix J: SHARP Investigators Study Group. Plasma biomarkers as predictors of outcome in patients with advanced hepatocellular carcinoma. Clin Cancer Res 18: 2290-2300, 2012.

14. Ogasawara S, Chiba T, Ooka Y, Suzuki E, Kanogawa N, Saito T, Motoyama T, Tawada A, Kanai F and Yokosuka O: Liver function assessment according to the Albumin-Bilirubin (ALBI) grade in sorafenib-treated patients with advanced hepatocellular carcinoma. Invest New Drugs 33: 1257-1262, 2015.

15. Choi GH, Shim JH, Kim MJ, Ryu MH, Ryoo BY, Kang YK, Shin YM, Kim KM, Lim YS and Lee HC: Sorafenib alone versus sorafenib combined with transarterial chemoembolization for advanced-stage hepatocellular carcinoma: Results of propensity score analyses. Radiology 269: 603-611, 2013. 
16. Kudo M, Matsui O, Izumi N, Kadoya M, Okusaka T, Miyayama S, Yamakado K, Tsuchiya K, Ueshima K, Hiraoka A, et al: Transarterial chemoembolization failure/refractoriness: JSH-LCSGJ criteria 2014 update. Oncology 87 (Suppl 1) S22-S31, 2014

17. Printz C: Clinical trials of note. Sorafenib as adjuvant treatment in the prevention of disease recurrence in patients with hepatocellular carcinoma (HCC) (STORM). Cancer 115: 4646, 2009.

18. Prado CM, Lieffers JR, McCargar LJ, Reiman T, Sawyer MB Martin L and Baracos VE: Prevalence and clinical implications of sarcopenic obesity in patients with solid tumors of the respiratory and gastrointestinal tracts: A population based study. Lancet Oncol 9: 629-635, 2008.

19. Rosenberg IH: Sarcopenia: Origins and clinical relevance. J Nutr 127: 990S-991S, 1997.

20. Wang C and Bai L: Sarcopenia in the elderly: Basic and clinical issues. Geriatr Gerontol Int 12: 388-396, 2012.

21. Dasarathy S: Consilience in sarcopenia of cirrhosis. J Cachexia Sarcopenia Muscle 3: 225-237, 2012.

22. Periyalwar P and Dasarathy S: Malnutrition in cirrhosis: Contribution and consequences of sarcopenia on metabolic and clinical responses. Clin Liver Dis 16: 95-131, 2012.

23. Nishikawa H, Yoh K, Enomoto H, Iwata Y, Kishino K, Shimono Y, Hasegawa K, Nakano C, Takata R, Nishimura T, et al: Factors associated with protein-energy malnutrition in chronic liver disease: Analysis using indirect calorimetry. Medicine (Baltimore) 95: e2442, 2016.

24. Fukushima H, Nakanishi Y, Kataoka M, Tobisu K and Koga F: Prognostic significance of sarcopenia in patients with metastatic renal cell carcinoma. J Urol 195: 26-32, 2016.

25. Levolger S, van Vugt JL, de Bruin RW and IJzermans JN Systematic review of sarcopenia in patients operated on for gastrointestinal and hepatopancreatobiliary malignancies. Br J Surg 102: 1448-1458, 2015

26. Kobayashi A, Kaido T, Hamaguchi Y, Okumura S, Taura K, Hatano E, Okajima $\mathrm{H}$ and Uemoto S: Impact of postoperative changes in sarcopenic factors on outcomes after hepatectomy for hepatocellular carcinoma. J Hepatobiliary Pancreat Sci 23 : 57-64, 2016.

27. Harimoto N, Shirabe K, Yamashita YI, Ikegami T, Yoshizumi T, Soejima Y, Ikeda T, Maehara Y, Nishie A and Yamanaka T: Sarcopenia as a predictor of prognosis in patients following hepatectomy for hepatocellular carcinoma. Br J Surg 100: $1523-1530,2013$

28. Takeda H, Nishikawa H, Osaki Y, Tsuchiya K, Joko K, Ogawa C, Taniguchi H, Orito E, Uchida Y and Izumi N; Japanese Red Cross Liver Study Group: Clinical features associated with radiological response to sorafenib in unresectable hepatocellular carcinoma: A large multicenter study in Japan. Liver Int 35: 1581-1589, 2015

29. Nishikawa H, Takeda H, Tsuchiya K, Joko K, Ogawa C, Taniguchi H, Orito E, Uchida Y, Osaki Y and Izumi N; Japanese Red Cross Liver Study Group: Sorafenib therapy for BCLC stage $\mathrm{B} / \mathrm{C}$ hepatocellular carcinoma; clinical outcome and safety in aged patients: A multicenter study in Japan. J. Cancer 5: 499-509, 2014

30. Cheng AL, Amarapurkar D, Chao Y, Chen PJ, Geschwind JF, Goh KL, Han KH, Kudo M, Lee HC, Lee RC, et al: Re-evaluating transarterial chemoembolization for the treatment of hepatocellular carcinoma: Consensus recommendations and review by an International Expert Panel. Liver Int 34: 174-183, 2014.
31. Fujiwara N, Nakagawa H, Kudo Y, Tateishi R, Taguri M, Watadani T, Nakagomi R, Kondo M, Nakatsuka $T$, Minami T, et al: Sarcopenia, intramuscular fat deposition, and visceral adiposity independently predict the outcomes of hepatocellular carcinoma. J Hepatol 63: 131-140, 2015.

32. Trotti A, Colevas AD, Setser A, Rusch V, Jaques D, Budach V, Langer C, Murphy B, Cumberlin R, Coleman CN and Rubin P: CTCAE v3.0: Development of a comprehensive grading system for the adverse effects of cancer treatment. Semin Radiat Oncol 13: 176-181, 2003.

33. European Association For The Study Of The Liver; European Organisation For Research And Treatment Of Cancer: EASL-EORTC Clinical Practice guidelines: Management of hepatocellular carcinoma. J Hepatol 56: 908-943, 2012.

34. Lencioni R and Llovet JM: Modified RECIST (mRECIST) assessment for hepatocellular carcinoma. Semin Liver Dis 30: $52-60,2010$.

35. Salvaggio G, Furlan A, Agnello F, Cabibbo G, Marin D, Giannitrapani L, Genco C, Midiri M, Lagalla R and Brancatelli G: Hepatocellular carcinoma enhancement on contrast-enhanced CT and MR imaging: Response assessment after treatment with sorafenib: Preliminary results. Radiol Med 119: 215-221, 2014.

36. Albers I, Hartmann H, Bircher J and Creutzfeldt W: Superiority of the Child-Pugh classification to quantitative liver function tests for assessing prognosis of liver cirrhosis. Scand J Gastroenterol 24: 269-276, 1989.

37. Saito M, Seo Y, Yano Y, Miki A, Yoshida M and Azuma T: Short-term reductions in non-protein respiratory quotient and prealbumin can be associated with the long-term deterioration of liver function after transcatheter arterial chemoembolization in patients with hepatocellular carcinoma. J Gastroenterol 47: 704-714, 2012.

38. Hanai T, Shiraki M, Ohnishi S, Miyazaki T, Ideta T, Kochi T, Imai K, Suetsugu A, Takai K, Moriwaki $\mathrm{H}$ and Shimizu M: Rapid skeletal muscle wasting predicts worse survival in patients with liver cirrhosis. Hepatol Res 46: 743-751, 2016.

39. Chindapasirt J: Sarcopenia in Cancer Patients. Asian Pac J Cancer Prev 16: 8075-8077, 2015.

40. Mir O, Coriat R, Blanchet B, Durand JP, Boudou-Rouquette P, Michels J, Ropert S, Vidal M, Pol S, Chaussade S and Goldwasser F: Sarcopenia predicts early dose-limiting toxicities and pharmacokinetics of sorafenib in patients with hepatocellular carcinoma. PLoS One 7: e37563, 2012

41. Shiraki M, Nishiguchi S, Saito M, Fukuzawa Y, Mizuta T, Kaibori M, Hanai T, Nishimura K, Shimizu M, Tsurumi $H$ and Moriwaki H: Nutritional status and quality of life in current patients with liver cirrhosis as assessed in 2007-2011. Hepatol Res 43: 106-112, 2013.

42. Prado CM, Wells JC, Smith SR, Stephan BC and Siervo M: Sarcopenic obesity: A Critical appraisal of the current evidence. Clin Nutr 31: 583-601, 2012.

43. McCurry J: Japan battles with obesity. Lancet 369: 451-452, 2007.

44. Examination Committee of Criteria for 'Obesity Disease' in Japan; Japan Society for the Study of Obesity: New criteria for 'obesity disease' in Japan. Circ J 66: 987-992, 2002. 